\title{
The effects of proton pump inhibitors on autonomic tone in patients with erosive and non-erosive esophagitis
}

\author{
E. L. JONES,* S. PERRING,* A. KHATTAB† \& O. ALLENBY-SMITH $\ddagger$ \\ *Medical Physics Department, Poole Hospital NHS Foundation Trust, Poole, UK \\ $\dagger$ Faculty of Health and Social Sciences, Bournemouth University, Bournemouth, UK \\ $\ddagger$ Department of Surgery, Poole Hospital NHS Foundation trust, Poole, UK
}

\begin{abstract}
Background: Reduction in autonomic tone as measured by heart rate variability (HRV) has been associated with various inflammatory conditions including reflux disease. The nature of and permanence of this damage have not been fully assessed

Methods: 14 individuals with non-erosive reflux disease (NERD) and 10 individuals with erosive reflux disease (ERD) as identified on endoscopy were assessed for HRV prior to starting a course of proton pump inhibitor (PPI) therapy and 8 weeks from the start of PPI therapy.

Key results: Reflux symptoms were significantly improved by PPI therapy ( $\mathrm{P}=0.001)$, with no significant difference in reflux symptoms between the NERD and ERD groups either before $(\mathrm{P}=0.45)$ or following therapy $(\mathrm{P}=0.17)$. The ERD group displayed reduced HRV prior to PPI therapy as compared with a non-symptomatic group. There was significant improvement of HRV resulting from PPI therapy in the ERD group as measured by inspiration/ expiration ratio on forced breathing $(\mathrm{P}=0.02)$, Valsalva ratio $(\mathrm{P}=0.03)$ and extended metronome guided breathing at 6 breaths per minute $(\mathrm{P}=0.03)$. While a similar pattern was seen in the NERD group, the effects were not as strong and did not reach statistical significance.
\end{abstract}

Conclusions and Inferences: The results are consistent with a growing body of evidence that cardiac autonomic neuropathy as measured by HRV is associated with gastro-esophageal 
reflux disease and also suggest that successful treatment of the inflammation can lead to reversal of the deterioration of autonomic tone associated with that inflammation.

Keywords: Autonomic function. Heart rate Variability. Esophagitis.

\section{Key Messages:}

- Take-Home Message: Inflammatory reflux disease is deleterious to autonomic function but the effects can be reversed by successful treatment with PPI therapy

- Aims: Establish if autonomic tone is adversely affected by inflammatory reflux disease and if autonomic tone responds to healing PPI therapy

- Basic Methodology: 14 volunteers with non-erosive esophagitis and 10 with erosive esophagitis were assessed for heart rate variability prior to and 8 weeks following starting PPI therapy

- Results: Erosive esophagitis volunteers had reduced HRV as compared with a group of volunteers with no history of reflux. Volunteers with erosive esophagitis displayed significantly improved HRV following PPI therapy. Volunteers with non-erosive esophagitis had less pronounced changes that did not reach statistical significance. 


\section{INTRODUCTION}

Symptoms of gastro-esophageal reflux disease (GERD) are very common, affecting $8 \%$ to $25 \%$ of the population of Europe, (1). Acid reflux causes a range of symptoms including heartburn, epigastric pain, retrosternal pain, belching, dysphagia and regurgitation $(1,2)$. Proton Pump Inhibitor (PPI) therapy is a common treatment for GERD related symptoms. Caro et al (3) performed a Meta-analysis of 53 studies involving acute PPI therapy and maintenance PPI therapy. These studies looked at the PPI healing rates for erosive esophagitis at eight weeks. All PPI's showed a greater degree of healing esophagitis at eight weeks than $\mathrm{H}_{2}$-receptor antagonist or placebo and all maintained similar rates of symptom control

Erosive esophagitis is inflammation of the lining of the esophagus resulting from GERD. Esophagitis is graded endoscopically according to its severity using the Los Angeles Classification (4). The majority of patients with reflux symptoms will have some esophagitis visible on endoscopy, described as erosive reflux disease (ERD). However a significant proportion of patients with significant reflux symptoms have no obvious esophagitis on endoscopy, this group being described as having non-erosive reflux disease (NERD) (5).

Cardiovascular autonomic function is known to be significantly and permanently compromised in a number of diseases, notably diabetes Parkinson's disease and multi-system atrophy (6). Significant autonomic failure is accompanied by a range of symptoms including dizziness, loss of consciousness, diarrhoea, excessive sweating and loss of sexual function (7). It is also associated with a large increase in risk of sudden cardiac death $(8,9)$. 
Previous research has shown that the performance of the autonomic nervous system is significantly attenuated in diabetics compared with a healthy age-matched cohort in the absence of typical symptoms of autonomic failure (10). Various studies have noted deterioration in autonomic regulation resulting from various inflammatory conditions including pneumonia (11), and even resulting from the minor inflammatory response to the influenza vaccine (12). Cardiovascular autonomic function appears to be adversely affected by reflux disease, though the extent of deterioration differs significantly between patients with and without erosive esophagitis (13).

This study aimed to examine autonomic tone in patients with mild erosive reflux (esophagitis) disease (ERD) and those with non-erosive reflux disease (NERD) and assess the change in autonomic tone in the two groups resulting from an eight week healing dose of PPI therapy to see if treatment for GERD can reverse any deterioration in autonomic tone observed.

\section{MATERIALS AND METHODS}

Patients aged 18 - 80 years old attending for routine gastroscopy (OGD) in the Endoscopy Department of Poole Hospital NHS Foundation Trust were considered for inclusion in this project and approached if they were considered to have a high clinical probability of GERD. This was assessed on the basis of having typical reflux symptoms or non-cardiac chest pain, have some endoscopic indication for reflux (GERD, lax sphincter or hiatus hernia) and their treatment plan following endoscopy to include a prescription for PPI therapy. Patients were excluded for consideration if they had severe (grade C or D) esophagitis or Barrett's Disease or any other upper GI pathology including gastritis, or if they had used PPI therapy for a 
significant time period. Patients were also excluded if they had Diabetes Mellitus, heart disease or any acute or chronic inflammatory condition apart from esophagitis.

We recruited 24 participants in total between September 2010 and November 2013, 14 having no endoscopically identifiable esophagitis (NERD group) and 10 with grade A or B esophagitis (ERD group). The demographic details are summarised in Table 1.

Volunteers were assessed for autonomic function prior to initiation of their PPI therapy and at least 8 weeks following initiation of PPI therapy. Volunteers completed a standard GERD impact questionnaire at the time of autonomic assessment on both visits (http://www.epgpatientdirect.org/docs/gerd/questionnaire_gerd-impact-scale[1].pdf).

Autonomic function testing was performed after the methods described in Perring and Jones (12). A ProComp Infiniti Biosignal Recorder (Thought Technology, Montreal, Canada) was used to collect ECG and chest wall plethysmography signals from the volunteers. Data was collected in real-time at $1024 \mathrm{~Hz}$ for the ECG data and $256 \mathrm{~Hz}$ for the chest wall plethysmography data. Data was transferred to a PC via an optical fibre interface and recorded using in-house developed software written in Visual Basic 6 (Microsoft Corporation).

The volunteers were required not to eat anything and to refrain from caffeine and nicotine for at least 3 hours prior to the assessment. Where possible the volunteers were re-assessed at the same time of day to minimise any diurnal variation. 
The following sequence of provocations was used to assess autonomic cardiovascular reflexes:

1 An extended rest period (5 min).

2 A trial period of at least two cycles of deep breathing at six breaths per min.

3 Valsalva Manoeuvre (open glottis, pressure achieved >20 mmHg, maintained for $10 \mathrm{~s}$ ).

4 Maximal hand grip (10 s squeeze without breath hold, repeated after $30 \mathrm{~s}$ ).

5 Two minutes of metronome-guided deep breathing at six breaths per minute.

6. Lying standing manoeuvre (ratio of heart rate at beat 15 following standing over heart rate at beat 30 following standing)

Analysis included the following:

1. Extended resting heart rate variability (SDNN, PNN50 and low frequency/ high frequency $(\mathrm{LF} / \mathrm{HF})$ ratio.

2. Analysis of the provocations originally proposed by Ewing et al (14) and detailed in Mathias and Bannister (15).

- Heart rate difference between maximum and minimum heart rate breathing at 5 seconds inhalation, 5 seconds exhalation at maximum depth, in beats per minute (bpm) (I/E Diff)

- Ratio of heart rate during maximal handgrip to heart rate during resting

- Ratio of maximum heart rate following standing (approximately 15 beats after standing) to minimum heart rate following standing (approximately 30 beats following standing)

3. Analysis of heart rate variability during 2 minutes of metronome guided breathing at 6 breaths per minute. Assessment of the correlation of heart rate and chest wall movement as well as amplitude of heart rate variation in temporal and frequency space, resulting in a 
combined parameter (DEV) representing the number of standard deviations outside of normal age matched values as described in Perring and Jones (12).

Ethical approval was sought and approval was given by Hampshire Research Ethics Committee (10/H0504/31)

\section{RESULTS}

\section{Comparison of Symptom Responses in the Two Sample Groups}

Analysis of the results of the GERD Impact scale using a paired t-test shows a dramatic statistical difference $(\mathrm{p}=<0.001)$ in the scores before and after eight weeks of PPI therapy, indicating a dramatic and consistent improvement in GERD symptoms resulting from PPI therapy. There was no significant difference between the symptom impact scores of the ERD and NERD groups, either before or after 8 weeks of PPI therapy $(\mathrm{P}=0.45$ and $\mathrm{P}=0.17$ respectively)

\section{Comparison of initial HRV in reflux group versus normal volunteer group}

Measurement parameters for the initial (non-treated) NERD/ERD groups were compared with data collected from 71 normal volunteers prior to influenza vaccine reported in a previous paper by Perring and Jones (12). There was no significant difference in age between the previous normal group of 71 individuals and the ERD, NERD or combined groups. The results of this data comparison using independent t-testing suggest that there does appear to be a statistically significant difference in many of the measures of HRV between a normal healthy volunteer group from a previous study and the ERD group, with the HRV parameters lower in the ERD group than the normal group (see table 2). There did appear to be a similar 
trend to lower HRV in the NERD group, but the differences with the normal group did not reach statistical significance in the majority of HRV parameters assessed.

\section{Comparison of HRV prior to and following PPI treatment}

Measurement parameters for assessment of HRV were compared prior to initiation of PPI treatment and following 8 weeks of PPI treatment for both the NERD and ERD groups. There was a generalised increase in HRV values for both groups between the two visits indicating an improvement in autonomic tone, but only for the ERD group was there statistically significant improvement for 3 parameters of HRV. Analysis of the data for the NERD group showed no significant change in autonomic tone after eight weeks of PPI therapy.

\section{DISCUSSION}

We remain circumspect about the weight of these results due to the small number of subjects recruited to both groups and the lack of consistent changes across the full range of parameters measured. However this being said, the study does still suggest a tendency for individuals with symptoms of reflux disease to have reduced HRV consistent with some reduction in autonomic tone as compared with a healthy group and that furthermore the effect is strongest in individuals with macroscopic inflammation identified on gastrography (ERD). This finding is in keeping with research by Lee et al (16) who found a difference in autonomic tone in the ERD group compared with the NERD group. Chen (13) also found that autonomic function appears to differ significantly between the two groups, with spectral analysis of heart rate variability significantly reduced in the high frequency power band in patients with erosive esophagitis. In contrast the reported symptom impact scores of the two groups did not significantly differ between the NERD and ERD groups at baseline assessment. 
The results of this study also suggest a significant improvement in several parameters of HRV following a healing treatment of PPI therapy in individuals with ERD. This statistically significant improvement in HRV was not observed in the NERD group. This is in spite of there being no difference between the reported symptom impact scores in the two groups following PPI therapy, indicating that both groups recorded a similar symptomatic benefit from the PPI therapy.

As a result of this study we concur with Lee et al (16) that "It is the structural state of the oesophagus and not the symptomology which dictates the autonomic function status.”

There is a growing weight of evidence that deterioration in autonomic reflexes is associated with inflammatory conditions, the suggested route of interaction being via the cholinergic anti-inflammatory pathway (17). This study further suggests that amelioration of the inflammatory condition, in this case by treatment of acid reflux, can reverse the deterioration in autonomic tone. There is clear evidence that deterioration in autonomic responses with type 2 diabetes is associated with significant symptoms and a rise in cardiovascular risk and risk of sudden death (18). It is at present unclear but entirely possible that minor deterioration in autonomic reflexes as are associated with reflux disease may be associated with a smaller but still raised risk of cardiac events.

Given the reversible nature of the HRV changes seen in this study it could be argued that there may be justification for the treatment of GERD patients, particularly those who are elderly and/or with diabetes, with anti-inflammatory medication alongside conventional PPI therapy to reduce the impact on the autonomic nervous system. 
This trial did not include individuals with severe inflammatory reflux disease (grades C or D esophagitis or Barrett's Disease), which we suspect would have more severe deterioration of autonomic tone and whose symptoms may in part be aggravated by symptoms associated with impaired autonomic reflexes.

\section{CONCLUSIONS}

This paper does add weight to other published evidence that mild esophagitis is associated with a minor deterioration in autonomic reflexes. Furthermore the results support the proposition that this mild deterioration in autonomic performance is reversed by treatment of esophagitis with PPI therapy.

\section{ACKNOWLEDGMENTS}

The project was supported by Medical Physics, Poole Hospital NHS Foundation Trust. Thanks to Nick Taylor (nurse endoscopist) for assisting with recruitment and for Theresa Royles for assisting with data collection.

\section{FUNDING}

The authors have no other funding to declare.

\section{DISCLOSURE}

The authors have no competing interests or disclosures to declare.

\section{AUTHOR CONTRIBUTION}

EJ designed the research study, performed the data collection, analyzed the data, and cowrote the paper; SP designed the research study, performed the data collection, assisted with 
analysis of the data, and co-wrote the paper; AK supervised the project and assisted with writing the paper; OAS recruited the majority of patients and assisted in writing the paper; NT assisted with recruitment; TR contributed to data collection.

\section{References}

1. Boeckxstaens G, El-Serag HB, Smout A, Kahrilas P. Symptomatic reflux disease: the present, the past and the future. Gut. 2014 Jul;63(7):1185-93.

2. Fass R, Wong W-M. Gastroesophageal reflux disease. In: Weinstein WM, Hawkey CJ, Bosch J, eds. Clinical Gastroenterology and Hepatology. Philadelphia: Elsevier Mosby 2005:157-166.

3. Caro J, Salas M and Ward A. Healing and relapse rates in gastroesophageal reflux disease treated with the newer proton-pump inhibitors lansoprazole, rabeprazole, and pantoprazole compared with omeprazole, ranitidine, and placebo: evidence from randomized clinical trials. Clinical Therapeutics 2001; 23 (7): 998-1017.

4. Lundell LR, Dent J, Bennett JR, Blum, AL, Armstrong D, Galmiche JP, Johnson F, Hongo M, et al. Endoscopic assessment of oesophagitis: clinical and functional correlates and further validation of the Los Angeles classification. Gut. 1999 Aug;45(2):172-80

5. Barlow WJ, Orlando RC. The pathogenesis of heartburn in nonerosive reflux disease: a unifying hypothesis. Gastroenterology. 2005 Mar;128(3):771-8.

6. Ziemssen T. Treatment of dysautonomia in extra pyramidal disorders. Therapeutical Advances in Neurological Disorders 2010; 3 (1): 53-67. 
7. Khurana RK. Dysautonomia. In: Schuster MM and Crowell MD and Koch KL. Atlas of Gastrointestinal Motility in Health and Disease. 2nd Edition. BC.Decker Publishing 2002: 335-352.

8. O’Brien IA, Mc Fadden JP, Corrall RJ. The influence of autonomic neuropathy on mortality in insulin dependent diabetes. Quarterly Journal of Medicine 1991; 79: 495-502.

9. Töyry JP, Niskanen LK, Mäntysaari MJ, Länsimies EA, Uusitupa MI. Occurrence, predictors, and clinical significance of autonomic neuropathy in NIDDM. Ten-year follow-up from the diagnosis. Diabetes 1996; 45(3):308-15

10. Perring S, Jones E. Simultaneous measurement of instantaneous heart rate and chest wall plethysmography in short-term, metronome guided heart rate variability studies: suitability for assessment of autonomic dysfunction. Physiolical Measurement 2003; 24:745-751.

11. Vassallo M, Allen SC. A study of autonomic cardiovascular reflexes in elderly patients with pneumonia. International Journal of Clinical Practice. 1997; 51(7), 438-41.

12. Perring S and Jones E. Assessment of changes in cardiac autonomic tone resulting from inflammatory response to the influenza vaccination. Clinical Physiology and Functional Imaging. 2012; 32: 437-444. 
13. Chen CL, Orr W, Yang C and Kuo T. Cardiac autonomic regulation differentiates reflux disease with and without erosive esophagitis. Scandinavian Journal of Gastroenterology. 2006; 41: 1001-1006

14. Ewing DJ, Martyn CN, Young RJ and Clarke BF. The value of cardiovascular autonomic function tests, 10 years of experience in diabetes. Diabetes Care. 1985; 8 (5): 491-98

15. Mathias C J and Bannister R. Autonomic Failure. A textbook of clinical disorders of the Autonomic Nervous System 4th Edition. Oxford Medical Publications, 2006.

16. Lee YC, Wang HP, Lin LY, Lee BC, Chiu HM, Wu MS, Chen MF, Lin JT. Heart rate variability in patients with different manifestations of gastroesophageal reflux disease. Autonomic Neuroscience. 2004 Nov 30;116(1-2):39-45

17. Czura CJ, Tracey KJ. Autonomic neural regulation of immunity. Journal of Internal Medicine. 2005; 257: 156-166.

18. Vinik AI, Erbas T, Casellini CM. Diabetic cardiac autonomic neuropathy, inflammation and cardiovascular disease. Journal of Diabetes Investigations. 2013; Jan;4(1):4-18.

Hongo M, Richter JE, S J Spechler SJ, Tytgat G, L Wallin L (1999) Endoscopic assessment of oesophagitis: clinical and functional correlates and further validation of the Los Angeles classification Gut. 45: 1672-180. 
Table 1. Demographic details of sample groups

\begin{tabular}{|l|l|l|l|}
\hline & Total & NERD & ERD \\
\hline Number of volunteers & 24 & 14 & 10 \\
\hline Age range & $26-72$ & $26-65$ & $42-72$ \\
\hline Age (mean+/-SD) & $50.1+/-11.3$ & $49.1(13.6)$ & $51.6(9.5)$ \\
\hline Gender M:F & $14: 10$ & $8: 5$ & $6: 5$ \\
\hline
\end{tabular}

Table 2. Comparison of initial HRV in reflux group versus normal volunteer group

\begin{tabular}{|c|c|c|c|c|c|}
\hline & $\begin{array}{l}\text { Value for } \\
\text { Normal Group } \\
\text { mean (SD) N=71 }\end{array}$ & $\begin{array}{l}\text { Value for ERD } \\
\text { Group } \\
\text { mean (SD) N=10 }\end{array}$ & t (P Val) & $\begin{array}{l}\text { Value for } \\
\text { NERD Group } \\
\text { mean (SD) } \\
\mathrm{N}=14\end{array}$ & t (P Val) \\
\hline Age (years) & 48.7 (11.9) & $51.2(9.5)$ & $0.6(0.55)$ & $50.8(13.6)$ & $0.54(0.58)$ \\
\hline \multicolumn{6}{|l|}{ Resting Measures } \\
\hline $\begin{array}{l}\text { Resting Heart Rate } \\
\text { (bpm) }\end{array}$ & 73.45 (10.91) & $70.6(10.01)$ & $0.65(0.52)$ & $67.1(9.8)$ & $1.19(0.06)$ \\
\hline SDNN (ms) & 46.66 (25.8) & $43.6(22.5)$ & $-0.18(0.86)$ & $53.2(34.5)$ & $-2.12(\mathbf{0 . 0 4 )}$ \\
\hline PNN50 (\%) & $8.80(11.61)$ & $3.5(2.9)$ & $3.3(\mathbf{0 . 0 2})$ & $3.7(6.1)$ & $-0.02(0.85)$ \\
\hline LF/HF Ratio & $1.06(0.07)$ & $3.6(1.6)$ & $-8.12(\mathbf{0 . 0 0 1 )}$ & $4.11(2.24)$ & $-9.95(\mathbf{0 . 0 0 1})$ \\
\hline
\end{tabular}




\begin{tabular}{|c|c|c|c|c|c|}
\hline \multicolumn{6}{|c|}{ Ewing Provocation Tests } \\
\hline I/E Diff (bpm) & $14.18(7.73)$ & $9.7(4.5)$ & $1.66 \mathbf{( 0 . 0 2 )}$ & $13.1(8.1)$ & $0.22(0.82)$ \\
\hline Valsalva (ratio) & $1.33(0.18)$ & $1.2(0.1)$ & $1.79(0.06)$ & $1.35(0.3)$ & $-0.79(0.43)$ \\
\hline Hand grip (ratio) & $1.17(0.09)$ & $1.1(0.1)$ & $1.33 \mathbf{( 0 . 0 5 )}$ & $1.1(0.1)$ & $1.51(0.13)$ \\
\hline \multicolumn{6}{|c|}{ Metronome Breathing Analysis } \\
\hline $\begin{array}{l}\text { DEV (SD's from age- } \\
\text { matched norm) }\end{array}$ & $0.29(1.2)$ & $-0.25(1.4)$ & $1.32(0.19)$ & $0.38(1.2)$ & $0.84(0.40)$ \\
\hline
\end{tabular}

Table 3 Changes in HRV parameters during Ewing assessment and metronome guide breathing for the ERD and NERD group prior to and following eight weeks on PPI therapy.

\begin{tabular}{|c|c|c|c|c|c|c|}
\hline & \multicolumn{3}{|c|}{ ERD Group (N=10) } & \multicolumn{3}{|c|}{ NERD Group (N=14) } \\
\hline & $\begin{array}{l}\text { Visit } 1 \\
\text { Mean } \\
( \pm S D)\end{array}$ & $\begin{array}{l}\text { Visit } 2 \\
\text { Mean } \\
( \pm S D)\end{array}$ & $\begin{array}{l}\text { t Val } \\
\text { (P Val) }\end{array}$ & $\begin{array}{l}\text { Visit } 1 \\
\text { Mean } \\
( \pm S D)\end{array}$ & $\begin{array}{l}\text { Visit } 2 \\
\text { Mean } \\
( \pm \text { SD) }\end{array}$ & $\begin{array}{l}\text { t Val } \\
\text { (P Val) }\end{array}$ \\
\hline \multicolumn{7}{|c|}{ GERD Impact Scale } \\
\hline Average scores & $12.2(4.6)$ & $3.3(3.9)$ & $5.9(0.001)$ & $13.7(4.8)$ & $5.3(4.7)$ & $7.7(0.001)$ \\
\hline \multicolumn{7}{|c|}{ Resting Measures } \\
\hline Resting HR (bpm) & $70.6(9.6)$ & 74.7 (10.1) & $2.3(0.06)$ & $67.1(9.8)$ & $67.3(10.1)$ & $0.8(0.43)$ \\
\hline SDNN (ms) & 43.6 (22.5) & $48.7(18.4)$ & $2.2(0.06)$ & $53.2(34.5)$ & $53.5(24.1)$ & $1.1(0.28)$ \\
\hline PNN50 (\%) & $3.5(2.9)$ & $2.86(2.84)$ & $1.9(0.08)$ & $3.7(6.1)$ & $2.8(2.7)$ & $1.9(0.08)$ \\
\hline LF/HF Ratio & $3.6(1.6)$ & 3.55 (1.8) & $1.4(0.18)$ & $4.11(2.24)$ & $4.13(2.27)$ & $0.8(0.44)$ \\
\hline \multicolumn{7}{|c|}{ Ewing Provocation Tests } \\
\hline I/E Diff (bpm) & $9.7(4.5)$ & $13.3(5.5)$ & $2.84(\mathbf{0 . 0 2})$ & $13.1(8.1)$ & $15.1(8.8)$ & $1.9(0.08)$ \\
\hline Valsalva (ratio) & $1.2(0.1)$ & $1.3(0.2)$ & 2.5 (0.03) & $1.35(0.3)$ & $1.38(0.2)$ & $1.17(0.26)$ \\
\hline Hand grip (ratio) & $1.1(0.1)$ & $1.1(0.1)$ & $0.1(0.91)$ & $1.1(0.1)$ & $1.15(0.1)$ & $1.5(0.15)$ \\
\hline
\end{tabular}


Jones. PPI's and Autonomic Tone in Esophagitis16

\begin{tabular}{|l|l|l|l|l|l|l|}
\hline Lying / Standing (ratio) & $1.0(0.1)$ & $1.1(0.3)$ & $2.1(0.06)$ & $1.1(0.16)$ & $1.0(0.38)$ & $0.2(0.81)$ \\
\hline \\
Metronome Breathing Analysis \\
\hline DEV & $-0.25(1.4)$ & $0.44(1.3)$ & $2.5(\mathbf{0 . 0 3})$ & $0.38(1.2)$ & $0.56(1.4)$ & $1.09(0.29)$ \\
\hline
\end{tabular}

